\title{
Attractors under perturbation and discretization
}

\author{
Lars Grüne \\ Fachbereich Mathematik \\ J.W. Goethe-Universität \\ Postfach 111932 \\ 60054 Frankfurt a.M., Germany \\ gruene@math.uni-frankfurt.de
}

\begin{abstract}
Using control theoretic techniques we give a necessary and sufficient condition for the convergence of attractors in one step discretizations of ordinary differential equations and obtain estimates for the resulting discretization error. The necessary and sufficient condition is based on a robustness property for an associated perturbed system, which is closely related to but slightly weaker than the input-to-state stability property well known in control theory.
\end{abstract}

\section{Introduction}

A basic result in the numerical analysis of dynamical systems states that the existence of an attractor for an ordinary differential equation (ODE) implies the existence of a nearby attracting set for the discrete time system induced by a numerical one-step discretization [6]. This attracting set, however, may not be an attractor. More precisely, even if a sequence of attractors $A_{h_{n}}$ for a sequence of time steps $h_{n} \rightarrow 0$ converges to some set $A$ this set might not be an attractor for the approximated system, see e.g. [2, Example (2.12)] for a very simple two-dimensional system illustrating this fact and the monograph [9] for a detailed discussion.

There are basically two ways in order to ensure convergence of the numerical attractors $A_{h_{n}}$ to a "real" attractor $A$. The first is by imposing certain structural conditions on the approximated system or on its attractor, and the second is by imposing conditions on the numerical systems, see e.g. [9, Chapter 7] for examples of both approaches.

In this paper we take the second approach. We associate suitable "inflated" perturbed systems to both the ODE and its one-step numerical approximation, and show that a suitable robustness condition on the numerical systems is equivalent to the convergence of the attractor sequence $A_{h_{n}}$. Furthermore, this approach allows an a priory estimate of the numerical error, i.e. the Hausdorff distance between $A_{h_{n}}$ and $A$, which turns out to depend on both the order of the numerical scheme and the robustness of the $A_{h_{n}}$. The robustness condi- tion used here is closely related to the input-to-state stability property well known in control theory, which is introduced for arbitrary compact attractors in [7].

Techniques of this kind have also been used in $[3,4]$. In contrast to these references, where the perturbed (or controlled) systems have been used as auxiliary systems, here we focus on these systems themselves and formulate our results accordingly. This allows a compact notation both for the statements and the proofs and an improvement of the quantitative estimates in [3] under weaker assumptions.

\section{Setup and preliminaries}

We consider the ordinary differential equation in $\mathbb{R}^{d}$

$$
\dot{x}=f(x)
$$

where $f: \mathbb{R}^{d} \rightarrow \mathbb{R}^{d}$ is assumed to satisfy $\|f(x)\| \leq M$ for all $x \in \mathbb{R}^{d}$ and $\|f(x)-f(y)\| \leq L\|x-y\|$ for all $x, y \in \mathbb{R}^{d}$ and constants $M, L>0$. (These global assumptions can easily be weakened since we are only interested in the behaviour on compact subsets of the state space.) The solutions of (2.1) with initial value $x_{0} \in \mathbb{R}^{d}$ for initial time $t_{0}=0$ will be denoted by $\varphi\left(t, x_{0}\right)$.

In order to provide a framework for numerical one-step approximations of (2.1) we fix some $h_{0}>0$ and consider difference equations for time steps $h \in\left(0, h_{0}\right]$

$$
x(t+h)=\Psi_{h}(x(t))
$$

where $t \in h \mathbb{Z}:=\{h k \mid k \in \mathbb{Z}\}$, and $\Psi_{h}: \mathbb{R}^{d} \rightarrow \mathbb{R}^{d}$ satisfies $\left\|x-\Psi_{h}(x)\right\| \leq h M$ and $\|\left(\Psi_{h}(x)-x\right)-\left(\Psi_{h}(y)-\right.$ $y)\|\leq h L\| x-y \|$.

A special case of (2.2) is the time- $h$ map of (2.1) given by

$$
x(t+h)=\Phi_{h}(x(t)):=\varphi(h, x(t)) .
$$

Note that $L$ and $M$ from (2.1) need to be slightly enlarged in order to meet the assumptions on (2.2). 
Our main object of interest are numerical one-step approximations of (2.1) (or, more precisely, of (2.3)) which we will denote by

$$
x(t+h)=\tilde{\Phi}_{h}(x(t)) .
$$

Here we assume that (2.4) is of type (2.2) and, in addition,

$$
\left\|\tilde{\Phi}_{h}(x)-\Phi_{h}(x)\right\| \leq K h^{p+1}
$$

for some $p \in \mathbb{N}$ and some $K>0$. The value $p$ is called the order of the scheme. Typical examples of these schemes are Runge-Kutta and Taylor schemes, which are described in any textbook on numerical methods for ordinary differential equations, see e.g. [1, 5, 8].

Each of these equations defines a (semi-)dynamical system either in continuous or discrete time, which we denote by $\varphi(t, \cdot), \Psi_{h}(t, \cdot), \Phi_{h}(t, \cdot)$ and $\tilde{\Phi}_{h}(t, \cdot)$. In what follows we will sometimes write $\Phi(t, \cdot)$ which can be either a continuous or a discrete time system, and set $\mathbb{T}=\mathbb{R}$ for continuous time and $\mathbb{T}=h \mathbb{Z}$ for discrete time systems, the proper meaning being clear from the context. Furthermore, we abbreviate $\mathbb{T}^{+}:=\{t \in \mathbb{T} \mid t>0\}$, and for subsets $B \subset \mathbb{R}^{d}$ we use the convention $\Phi(t, B)=$ $\bigcup_{x \in B}\{\Phi(t, x)\}$.

We recall the definition of several distances between compact sets which will be used in what follows.

Definition 2.1 Let $C, D \subset \mathbb{R}^{d}$ be nonempty compact sets, $x \in \mathbb{R}^{d}$, and let $d$ be the Euclidean metric on $\mathbb{R}^{d}$. We define the distance from a point to a set by

$$
d(x, D):=\min _{y \in D} d(x, y),
$$

the nonsymmetric Hausdorff distance between two compact sets by

$$
\operatorname{dist}(C, D):=\max _{x \in C} \min _{y \in D} d(x, y),
$$

and the Hausdorff metric for compact sets by

$$
d_{H}(C, D):=\max \{\operatorname{dist}(C, D), \operatorname{dist}(D, C)\} .
$$

For $\varepsilon>0$ we denote the $\varepsilon$-ball around $C$ by $\mathcal{B}(\varepsilon, C):=$ $\left\{y \in \mathbb{R}^{d} \mid d(y, C)<\varepsilon\right\}$. If $C=\{x\}$ we also write $\mathcal{B}(\varepsilon, x)$.

Now we can define our objects of interest.

Definition 2.2 Let $\Phi=\varphi$ or $\Phi=\Psi_{h}$ for some $h>0$.

A compact set $B \subset \mathbb{R}^{d}$ is called forward invariant, if $\Phi(t, B) \subseteq B$ for all $t \in \mathbb{T}^{+}$and invariant, if $\Phi(t, B)=B$ for all $t \in \mathbb{T}^{+}$.

Given a compact forward invariant set $A \subset \mathbb{R}^{d}$ and a compact set $B \subset \mathbb{R}^{d}$ with $A \subset \operatorname{int} B$ we call $A$ attracting with attracted neighbourhood $B$, if

$$
\operatorname{dist}(\Phi(t, B), A) \rightarrow 0
$$

as $t \rightarrow \infty$.

$A$ is called an attractor (with attracted neighbourhood $B$ ) if it is invariant and attracting with attracted neighbourhood $B$.

Note that $A$ is a local attractor here, i.e. it is not assumed that each compact set $B \subset \mathbb{R}^{d}$ is an attracted neighbourhood.

Remark 2.3 It is easy to see that $A$ is an attractor with attracted neighbourhood $B$ if and only if it is the minimal forward invariant attracting set (with respect to set inclusion) with attracted neighbourhood $B$, cf. [3, Lemma 3.1]. In particular, if there exists an attractor $A$ with attracted neighbourhood $B$ then it is unique. Furthermore, any invariant set $D \subset$ intB is contained in $A$, cf. [3, Lemma 3.2].

\section{The inflated systems}

For the differential and difference equations under consideration we consider the following $\alpha$-perturbed systems (cf. $[3,4])$

$\dot{x}(t)=f(x(t))+\alpha u(t), \quad x(t+h)=\Psi_{h}(x(t))+\alpha h u_{h}(t)$

with solutions $\varphi^{\alpha}(t, x, u(\cdot))$ and $\Psi_{h}^{\alpha}\left(t, x, u_{h}(\cdot)\right)$, where $u(\cdot) \in \mathcal{U}$ and $u_{h}(\cdot) \in \mathcal{U}_{h}$ with $\mathcal{U}:=\{u: \mathbb{R} \rightarrow$ $\mathcal{B}(1,0) \mid u$ measurable $\}$ and $\mathcal{U}_{h}:=\left\{u_{h}: h \mathbb{Z} \rightarrow \mathcal{B}(1,0)\right\}$. (Recall that $\mathcal{B}(1,0)$ is the ball with radius 1 around the origin in $\mathbb{R}^{d}$ ). The set valued maps

$$
\begin{gathered}
\varphi^{\mathrm{infl}}(t, x, \alpha):=\bigcup_{u(\cdot) \in \mathcal{U}} \varphi^{\alpha}(t, x, u(\cdot)), \\
\Psi_{h}^{\mathrm{infl}}(t, x, \alpha):=\bigcup_{u_{h}(\cdot) \in \mathcal{U}_{h}} \Psi_{h}^{\alpha}\left(t, x, u_{h}(\cdot)\right)
\end{gathered}
$$

which to each point $x \in \mathbb{R}^{d}$ assign the reachable set from this point are called the $\alpha$-inflated dynamics.

Again for each $B \subset \mathbb{R}^{d}$ we define $\varphi^{\text {infl }}(t, B, \alpha):=$ $\bigcup_{x \in B} \varphi^{\text {infl }}(t, x, \alpha)$, and analogously for $\Psi_{h}^{\text {infl }}$. The following elementary Lemma follows immediately from the Gronwall Lemma, cf. [4, Lemma 10.1].

Lemma 3.1 Let $B \subset \mathbb{R}^{d}$ be a compact set and $h>0$. Consider the continuous time system $\varphi$ and its time- $h$ map $\Phi_{h}$. Then the inclusion

$$
\varphi^{\mathrm{infl}}(t, B, \alpha) \subseteq \Phi^{\mathrm{infl}}\left(t, B, e^{L h} \alpha\right)
$$

holds for all $t \in h \mathbb{N}$ and the constant $L$ from the Lipschitz estimate for (2.1). 
As for the unperturbed systems we can now define attracting sets.

Definition 3.2 Fix some $\alpha>0$ and let $\Phi^{\text {infl }}=\varphi^{\text {infl }}$ or $\Phi^{\mathrm{infl}}=\Psi_{h}^{\mathrm{infl}}$ for some $h>0$.

A compact set $B \subset \mathbb{R}^{d}$ is called $\alpha$-forward invariant, if $\Phi^{\text {infl }}(t, B, \alpha) \subseteq B$ for all $t \in \mathbb{T}^{+}$.

Given a compact $\alpha$-forward invariant set $A \subset \mathbb{R}^{d}$ and a compact set $B \subset \mathbb{R}^{d}$ with $A \subset \operatorname{int} B$ we call $A \alpha$ attracting with $\alpha$-attracted neighbourhood $B$, if

$$
\operatorname{dist}\left(\Phi^{\mathrm{infl}}(t, B, \alpha), A\right) \rightarrow 0
$$

as $t \rightarrow \infty$.

Now we formulate the robustness condition which will be used to obtain the convergence property. As usual we call a function $\gamma: \mathbb{R}_{0}^{+} \rightarrow \mathbb{R}_{0}^{+}$of class $\mathcal{K}_{\infty}$ if it is continuous, unbounded, strictly increasing, and satisfies $\gamma(0)=0$.

Definition 3.3 Let $\Phi=\varphi$ and $\Phi^{\text {infl }}=\varphi^{\text {infl }}$, or $\Phi=\Psi_{h}$ and $\Phi^{\text {infl }}=\Psi_{h}^{\text {inf }}$ for some $h>0$. Let $A \subset \mathbb{R}^{d}$ be an attracting set for $\Phi$ with attracted neighbourhood $B \subset \mathbb{R}^{d}$, let $\alpha^{*}>0$, and let $\gamma: \mathbb{R}_{0}^{+} \rightarrow \mathbb{R}_{0}^{+}$be a class $\mathcal{K}_{\infty}$ function.

Then we call $A$ (locally) $\gamma$-robust on $\left[0, \alpha^{*}\right]$, if for each $\alpha \in\left[0, \alpha^{*}\right]$ there exists an $\alpha$-attracting set $A_{\alpha}$ with $\alpha$ attracted neighbourhood $B$ for $\Phi^{\text {infl }}$ with

$$
d_{H}\left(A_{\alpha}, A\right) \leq \gamma(\alpha) .
$$

Note that there are different ways to express $\gamma$-robustness of attracting sets avoiding the explicit use of the associated inflated systems, see e.g. [3, 4]. A very related notion of robustness is the (local) input-to-state stability (LISS) which is introduced in [7] for compact attractors. Translated to our system it requires the existence of some $\rho>0$, some class $\mathcal{K}_{\infty}$ function $\gamma$ and some class $\mathcal{K} \mathcal{L}$ function $\beta$ (i.e. a function of two positive real arguments which is of class $\mathcal{K}_{\infty}$ in the first and decreasing to 0 in the second argument) such that for all $x \in \mathbb{R}^{d}$ with $d(x, A)<\rho$ and all $u(\cdot) \in \mathcal{U}$ with $\|u\|_{\infty}<\rho$ the inequality

$$
d\left(\varphi^{1}(t, x, u(\cdot)), A\right) \leq \beta(d(x, A), t)+\gamma\left(\|u(\cdot)\|_{\infty}\right)
$$

holds for all $t \geq 0$.

It is easily seen that (3.5) implies the robust attraction condition from Definition 3.3 with the same $\gamma$. The converse implication, however, is not true in general, i.e. Definition 3.3 does in general not imply (3.5) with the same $\gamma$ (although qualitatively the two properties describe the same behaviour as the different characterizations derived in [7] show). This is due to the fact, that (3.5) demands a uniform rate of attraction $\beta$ for all amplitudes of the perturbation $u(\cdot)$, whereas in Definition 3.3 the rate of attraction to $A_{\alpha}$ might depend on the perturbation amplitude $\alpha$, and hence attraction might become arbitrary slow as $\alpha \rightarrow 0$.

Remark 3.4 Note that each attractor $A$ is $\gamma$-robust on some interval $\left[0, \alpha^{*}\right]$ for suitable $\gamma$ of class $\mathcal{K}_{\infty}$ and $\alpha^{*}>0$, which essentially follows from the Lyapunov function argument in [6] or [9], see [3, Remark 2.6] for details.

Remark 3.5 If $A_{1}$ is some compact $\alpha$-attracting set with attracted neighbourhood $B$ for $\varphi^{\text {infl }}$ and some $\alpha>$ 0 , and $A_{2} \subset A_{1}$ then it is easily seen that the set $A$ defined by

$$
A:=\bigcap_{T>0} \bigcup_{t \geq T} \varphi^{\mathrm{infl}}(t, B, \alpha) \cup \bigcup_{t \geq 0} \varphi^{\mathrm{infl}}\left(t, A_{2}, \alpha\right)
$$

is $\alpha$-forward invariant, $\alpha$-attracting and satisfies $A_{2} \subseteq$ $A \subseteq A_{1}$.

\section{Main results}

With the help of the robustness condition from Definition 3.3 we can now formulate our main results.

Theorem 4.1 Consider a positive sequence of time steps $h_{n} \rightarrow 0$ as $n \rightarrow \infty$. Let $A_{h_{n}}$ be attractors for the numerical scheme $\tilde{\Phi}_{h_{n}}$ with attracted neighbourhood $B$ and assume that there exists some compact set $A \subset \operatorname{int} B$ such that

$$
\lim _{n \rightarrow \infty} d_{H}\left(A, A_{h_{n}}\right)=0 .
$$

Then $A$ is an attractor for the continuous time system (2.1) if and only if there exists a function $\gamma$ of class $\mathcal{K}_{\infty}$, a sequence $C_{n} \rightarrow 1$, some $\alpha^{*} \geq 0$ and some $n_{0} \in \mathbb{N}$ such that for all $n \geq n_{0}$ there exist $\gamma\left(C_{n}\right.$.)-robust attracting sets $\tilde{A}_{h_{n}} \supseteq A_{h_{n}}$ on $\left[0, \alpha^{*}\right]$ for $\tilde{\Phi}_{h_{n}}$ with $d_{H}\left(\tilde{A}_{h_{n}}, A_{h_{n}}\right) \rightarrow$ 0 as $n \rightarrow \infty$. In this case $A$ is $\gamma$-robust.

Note that it is not necessary that the discrete time attractors $A_{h_{n}}$ themselves are $\gamma\left(C_{n} \cdot\right)$-robust. If, however, we assume robustness of the $A_{h_{n}}$ then we can improve the result.

Theorem 4.2 Consider a positive sequence $h_{n} \rightarrow 0$ as $n \rightarrow \infty$, a function $\gamma$ of class $\mathcal{K}_{\infty}$ and some $\alpha^{*}>0$. Assume the existence of $\gamma$-robust attractors $A_{h_{n}}$ on $\left[0, \alpha^{*}\right]$ with attracted neighbourhood $B$ for the numerical scheme $\tilde{\Phi}_{h_{n}}$ and let $A \subset \operatorname{int} B$ be a compact set. Then the following four statements are equivalent. 
i) $A$ is an attractor with attracted neighbourhood $B$ for the continuous time system (2.1).

ii) $A$ is a $\gamma$-robust attractor with attracted neighbourhood $B$ for the continuous time system (2.1).

iii) $d_{H}\left(A_{h_{n}}, A\right) \rightarrow 0$ as $n \rightarrow \infty$.

iv) $d_{H}\left(A_{h_{n}}, A\right) \leq \gamma\left(K h_{n}^{p}\right)$ for all $h_{n}$ sufficiently small.

\section{Proofs}

We start with the following Lemma about $\gamma$-robustness under perturbations which will be crucial for proving the Theorems.

Lemma 5.1 Consider $h>0$ and two discrete time system $\Psi_{h}, \tilde{\Psi}_{h}$ of type $(2.2)$ such that

$$
\tilde{\Psi}_{h}(t, x) \in \Psi_{h}^{\mathrm{infl}}\left(t, x, \alpha_{0}\right)
$$

for all $t \in h \mathbb{N}$ and some $\alpha_{0}>0$. Assume that $A \subset \mathbb{R}^{d}$ is a $\gamma$-robust attracting set for $\Psi_{h}$ with attracted neighbourhood $B$ on $\left[0, \alpha^{*}\right]$ for some $\alpha^{*}>\alpha_{0}$. Then for each $D \in\left(1, \alpha^{*} / \alpha_{0}\right)$ there exists a compact set $A_{D}$ which is $\gamma(\cdot D /(D-1))$-robustly attracting with attracted neighbourhood $B$ on $\left[0, \alpha^{*}-\alpha_{0}\right]$ for $\tilde{\Psi}_{h}$ and satisfies $d_{H}\left(A_{D}, A\right) \leq \gamma\left(D \alpha_{0}\right)$.

Proof: Since $A$ is $\gamma$-robustly attracting on $\left[0, \alpha^{*}\right]$ for each $\alpha \in\left[0, \alpha^{*}\right]$ we find an $\alpha$-attracting set $A_{\alpha}$ for $\Psi_{h}^{\mathrm{infl}}$. Without loss of generality we may assume $A_{\alpha} \subseteq A_{\alpha^{\prime}}$ for all $\alpha \leq \alpha^{\prime}$. Now note that due to (5.6) $A_{\alpha_{0}+\beta}$ is $\beta^{\prime}$-attracting for $\Psi_{h}^{\mathrm{inf}}$ for each $\beta^{\prime} \in[0, \beta]$. Hence it is sufficient to show that there exists a mapping $b$ : $\left[0, \alpha^{*}-D \alpha_{0}\right] \rightarrow\left[\alpha_{0}, \alpha^{*}\right]$ such that $b(\beta) \geq \alpha_{0}+\beta, b(0) \leq$ $D \alpha_{0}$ and $d_{H}\left(A_{b(0)}, A_{b(\beta)}\right) \leq \gamma(\beta D /(D-1))$. Setting $A_{D}=A_{b(0)}$ then yields the assertion. We define $b$ as

$$
b(\beta):= \begin{cases}D \alpha_{0}, & \beta \in\left[0,(D-1) \alpha_{0}\right] \\ \alpha_{0}+\beta, & \beta \in\left[(D-1) \alpha_{0}, \alpha^{*}-\alpha_{0}\right]\end{cases}
$$

A simple calculation shows the desired estimates on $b$. In order to see the desired Hausdorff distance for $\beta \leq$ $(D-1) \alpha_{0}$ there is nothing to show. For $\beta \geq(D-1) \alpha_{0}$ we obtain $\alpha_{0}+\beta \leq \beta D /(D-1)$ which implies

$$
\begin{aligned}
d_{H}\left(A_{b(0)}, A_{b(\beta)}\right) & \leq d_{H}\left(A, A_{b(\beta)}\right) \leq \gamma(b(\beta)) \\
& \leq \gamma(\beta D /(D-1))
\end{aligned}
$$

and thus the desired estimate.

The next Lemma, which is proved in [3], provides a link between attractors of $\varphi$ and its time- $h$ map $\Phi_{h}$. As the statement might seem surprising at a first glance we restate the proof here.
Lemma 5.2 Let $h>0$ and $A_{h}$ be an attractor with attracted neighbourhood $B$ for the time- $h$ map $\Phi_{h}$ of the continuous time system (2.1). Then $A_{h}$ is also an attractor with attracted neighbourhood $B$ for system (2.1).

Proof: We first show invariance of $A_{h}$ for $\varphi$, i.e. $\varphi\left(t, A_{h}\right) \subseteq A_{h}$ for each $t \in \mathbb{R}$. By invariance of $A_{h}$ for $\Phi_{h}$ we know $\Phi_{h}\left(\varphi\left(t, A_{h}\right)\right)=\varphi\left(t, \Phi_{h}\left(A_{h}\right)\right)=\varphi\left(t, A_{h}\right)$, hence $\varphi\left(t, A_{h}\right)$ is invariant for $\Phi_{h}$, and by Remark 2.3 it is contained in $A_{h}$.

Thus we can conclude $\varphi\left(t, A_{h}\right) \subseteq A_{h}$ for each $t \in \mathbb{R}$, hence also $A_{h}=\varphi\left(-t, \varphi\left(t, A_{h}\right)\right) \subseteq \varphi\left(-t, A_{h}\right)$ for each $t \in \mathbb{R}$ and consequently $A_{h}$ is invariant for $\varphi$.

It remains to show the attractivity of $A$. Invariance of $A$ and continuous dependence on the initial value imply that for each $\delta>0$ there exists $\varepsilon>0$ such that

$$
d_{H}(D, A)<\varepsilon \quad \Rightarrow \quad d_{H}(\varphi(t, D), A)<\delta
$$

for all $t \in[0, h]$ and all $D \subset \mathbb{R}^{d}$. Since attractivity of $A$ for $\Phi_{h}$ implies $\lim _{i \rightarrow \infty, i \in \mathbb{N}} \operatorname{dist}(\varphi(i h, B), A)=0$ we can thus conclude $\lim _{t \rightarrow \infty} \operatorname{dist}(\varphi(t, B), A)=0$, i.e. $A$ is also an attracting set for $\varphi$ with attracted neighbourhood $B$, thus an attractor.

We now turn to the proofs of the main theorems.

Proof of Theorem 4.1: Assume the existence of the $\tilde{A}_{h_{n}}$ as stated in the Theorem. We first show that $A$ is $\gamma$-robustly attracting for $\varphi$ with attracted neighbourhod $B$. In order to prove this property, let $\alpha_{n}:=K h_{n}^{p}$. Then it is immediate from the definition of the discrete inflated dynamics and the assumptions on the one-step scheme that the time- $h_{n}$ map $\Phi_{h_{n}}$ satisfies

$$
\Phi_{h_{n}}(t, x) \in \tilde{\Phi}_{h}^{\mathrm{infl}}\left(t, x, \alpha_{n}\right)
$$

for all $t \in h_{n} \mathbb{N}$. Setting $D_{n}:=\sqrt{\alpha_{n}}$ and applying Lemma 5.1 for each $n>0$ with $\sqrt{\alpha_{n}} \leq \alpha^{*}$ and $D=D_{n}$ we obtain the existence of $\gamma\left(\cdot C_{n} D_{n} /\left(D_{n}-1\right)\right)$-robustly attracting sets $\bar{A}_{n}$ for the time- $h_{n}$ map $\Phi_{h_{n}}$ with

$$
d_{H}\left(\tilde{A}_{h_{n}}, \bar{A}_{n}\right) \leq \gamma\left(C_{n} D_{n} \alpha_{n}\right)
$$

In particular this implies

$$
d_{H}\left(\bar{A}_{n}, A\right) \rightarrow 0
$$

as $n \rightarrow \infty$. This immediately implies

$$
\operatorname{dist}(\varphi(t, B), A) \rightarrow 0,
$$

i.e. $A$ is indeed an attracting set with attracted neighbourhood $B$. In order to see the $\gamma$-robustness fix some $\alpha \in\left[0, \alpha^{*}\right)$. We have to show the existence of an $\alpha$-attracting set $A_{\alpha}$ for $\varphi^{\text {infl }}$ with $d_{H}\left(A_{\alpha}, A\right) \leq$ $\gamma(\alpha)$. Now first observe that by Lemma 3.1 for 
any $e^{L h_{n}} \alpha$-attracting set $B_{n}$ for $\Phi_{h_{n}}^{\text {inf }}$ the set $\bar{B}_{n}:=$ $\bigcup_{t \in\left[0, h_{n}\right]} \varphi^{\mathrm{infl}}\left(t, B_{n}, \alpha\right)$ is $\alpha$-attracting for $\varphi^{\mathrm{infl}}$ and satisfies $d_{H}\left(\bar{B}_{n}, B_{n}\right) \leq M h_{n}$ for the bound $M$ on $\|f\|$. Hence the $\gamma\left(\cdot C_{n} D_{n} /\left(D_{n}-1\right)\right)$-robustness of the sets $\bar{A}_{n}$ implies that for each $\varepsilon>0$ there exists an $\alpha$-attracting set $A_{\varepsilon}$ for $\varphi^{\text {infl }}$ with

$$
d_{H}\left(A_{\varepsilon}, A\right) \leq \gamma(\alpha)+\varepsilon
$$

This implies that

$$
\operatorname{dist}\left(\bigcup_{t \geq T} \varphi^{\operatorname{infl}}(t, B, \alpha), A\right) \leq \gamma(\alpha)+\varepsilon
$$

for all $T>0$ sufficiently large and

$$
\operatorname{dist}\left(\bigcup_{t \geq 0} \varphi^{\mathrm{infl}}(t, A, \alpha), A\right) \leq \gamma(\alpha)
$$

Thus by Remark 3.5 the set

$$
A_{\alpha}=\bigcap_{T>0} \bigcup_{t \geq T} \varphi^{\mathrm{infl}}(t, B, \alpha) \cup \bigcup_{t \geq 0} \varphi^{\mathrm{infl}}(t, A, \alpha)
$$

is $\alpha$-attracting, $\alpha$-forward invariant and satisfies

$$
d_{H}\left(A_{\alpha}, A\right) \leq \gamma(\alpha)
$$

i.e. the desired properties.

It remains to show that $A$ is an attractor, i.e. that $A$ is invariant for $\varphi$. If $A$ is not invariant, then by Remark 2.3 there exists an attractor $\bar{A} \subset A, \bar{A} \neq A$, with attracted neighbourhood $B$ for $\varphi$. By Remark 3.4 we can conclude that $\bar{A}$ is $\bar{\gamma}$-robust for a suitable $\bar{\gamma}$ of class $\mathcal{K}_{\infty}$. Hence by Lemma 5.1 for $\varepsilon>0$ sufficiently small and all $n>0$ sufficiently large there exist attracting sets $\hat{A}_{n}$ for the numerical schemes $\tilde{\Phi}_{h_{n}}$ with $\operatorname{dist}\left(A, \hat{A}_{h_{n}}\right)>\varepsilon$. Again by Remark 2.3 we can conclude $A_{h_{n}} \subseteq \hat{A}_{h_{n}}$. This implies $\operatorname{dist}\left(A, A_{h_{n}}\right)>\varepsilon$, hence for all $n \in \mathbb{N}$ sufficiently large $\operatorname{dist}\left(\tilde{A}_{h_{n}}, A_{h_{n}}\right)>\varepsilon / 2$ which contradicts the assumption on the $\tilde{A}_{h_{n}}$.

For the converse assertion of the theorem, let $A$ be an attractor of $\varphi$. Then by Remark 3.4 there exists a function $\gamma$ of class $\mathcal{K}_{\infty}$ and some $\tilde{\alpha}^{*}>0$ such that $A$ is $\gamma$-robust on $\left[0, \tilde{\alpha}^{*}\right]$. As the same holds for each time- $h_{n}$ $\operatorname{map} \Phi_{h_{n}}, n \in \mathbb{N}$, Lemma 5.1 with $h=h_{n}, \Psi_{h}=\Phi_{h_{n}}$, $\tilde{\Psi}_{h}=\tilde{\Phi}_{h_{n}}, \alpha=K h_{n}^{p}$ and $D=1 / \sqrt{\alpha}$ immediately implies the assertion for all $n \in \mathbb{N}$ sufficiently large.

Proof of Theorem 4.2: The implications "(iv) $\Rightarrow$ (iii)" and "(ii) $\Rightarrow$ (i)" are obvious and "(iii) $\Rightarrow$ (ii)" follows immediately from Theorem 4.1. We now show "(ii) $\Rightarrow$ (iv)" and "(i) $\Rightarrow$ (iii)" implying the equivalence of the statements.

"(ii) $\Rightarrow$ (iv)": Since $A$ is also a $\gamma$-robust attractor for each time- $h_{n}$ map $\Phi_{h_{n}}$ we can conclude by Lemma 5.1 with $\alpha=K h_{n}^{p}$ that for each $D>1$ there exists an attracting set $A_{D}$ with $\operatorname{dist}\left(A_{D}, A\right) \leq \gamma\left(D K h_{n}^{p}\right)$. Since, furthermore, by Remark 2.3 we have $A_{h_{n}} \subseteq A_{D}$ for all $D>1$ we can conclude that

$$
\operatorname{dist}\left(A_{h_{n}}, A\right) \leq \gamma\left(K h_{n}^{p}\right) .
$$

Conversely, again by Lemma 5.1 for each $h_{n}$ and each $D>1$ we obtain the existence of an attracting set $\bar{A}_{h_{n}}^{D}$ for the time- $h_{n}$ map $\Phi_{h_{n}}$ with $\operatorname{dist}\left(\bar{A}_{h_{n}}^{D}, A_{h_{n}}\right) \leq$ $\gamma\left(D K h_{n}^{p}\right)$. Hence there is an attractor of $\Phi_{h_{n}}$ contained in $\bar{A}_{h_{n}}^{D}$ for each $D>1$, which by Lemma 5.2 coincides with $A$. This implies $A \subset \bar{A}_{h_{n}}^{D}$ and consequently

$$
\operatorname{dist}\left(A, A_{h_{n}}\right) \leq \gamma\left(D K h_{n}^{p}\right)
$$

for all $D>1$ which implies $\operatorname{dist}\left(A, A_{h_{n}}\right) \leq \gamma\left(K h_{n}^{p}\right)$ and thus finishes the proof of (iv).

"(i) $\Rightarrow$ (iii)" By Remark 3.4 there exists a class $\mathcal{K}_{\infty}$ function $\tilde{\gamma}$ such that $A$ is $\tilde{\gamma}$-robust. Without loss of generality we may assume $\tilde{\gamma} \geq \gamma$. Hence also the $A_{h_{n}}$ are $\tilde{\gamma}$-robust, and by the same arguments as for "(ii) $\Rightarrow$ (iv)", above, we obtain

$$
d_{H}\left(A, A_{h_{n}}\right) \leq \tilde{\gamma}\left(K h_{n}^{p}\right)
$$

implying (iii).

\section{References}

[1] P. Deuflhard and F. Bornemann. Numerische Mathematik. II: Integration gewöhnlicher Differentialgleichungen. de Gruyter, Berlin, 1994.

[2] B. G. Garay and P. E. Kloeden. Discretization near compact invariant sets. Random Comput. Dyn., 5:93-12, 1997.

[3] L. Grüne. Persistence of attractors for one-step discretizations of ordinary differential equations. DFGSchwerpunktprogramm "Dynamik: Analysis, effiziente Simulation und Ergodentheorie", Preprint 41/99, 1999. Submitted.

[4] L. Grüne. Convergence rates of perturbed attracting sets with vanishing perturbation. J. Math. Anal. Appl., 244:369-392, 2000.

[5] A. Iserles. A first course in the numerical analysis of differential equations. Cambridge Texts in Applied Mathematics. Cambridge University Press, 1995.

[6] P. E. Kloeden and J. Lorenz. Stable attracting sets in dynamical systems and their one-step discretizations. SIAM J. Numer. Anal., 23:986-995, 1986.

[7] E.D. Sontag and Y. Wang. New characterizations of input to state stability. IEEE Trans. Autom. Control, 41:1283-1294, 1996.

[8] J. Stoer and R. Bulirsch. Introduction to Numerical Analysis. Springer Verlag, New York, 1980.

[9] A. M. Stuart and A. R. Humphries. Dynamical Systems and Numerical Analysis. Cambridge University Press, 1996. 